\title{
Construction and Test of Low Cost X-Ray Tomography Scanner for Physical-Chemical Analysis and Nondestructive Inspections
}

\author{
José Martins de Oliveira Jr. ${ }^{a}$ and Antonio César Germano Martins ${ }^{b}$ \\ ${ }^{a}$ Universidade de Sorocaba-UNISO, Campus Seminário, Caixa Postal 578 , \\ Av. Dr. Eugênio Salermo, 100, Centro, 18035-430, Sorocaba, SP, Brazil \\ ${ }^{b}$ Universidade Estadual Paulista Julio de Mesquita Filho - UNESP, GASI, Av. 3 de Março, 511, Alto da \\ Boa Vista, 18087-180, Sorocaba, SP, Brazil.
}

\begin{abstract}
X-ray computed tomography (CT) refers to the cross-sectional imaging of an object measuring the transmitted radiation at different directions. In this work, we describe the development of a low cost micro-CT X-ray scanner that is being developed for nondestructive testing. This tomograph operates using a microfocus X-ray source and contains a silicon photodiode as detectors. The performance of the system, by its spatial resolution, has been estimated through its Modulation Transfer Function - MTF and the obtained value at 10\% of MTF is $661 \mu \mathrm{m}$. It was built as a general purpose nondestructive testing device.
\end{abstract}

Keywords: X-ray, Tomography, Nondestructive inspections, Image analysis PACS: 81.70.Tx; 82.80.Ej; 87.59.-e; 87.57.C-; 87.57.Q-

\section{INTRODUCTION}

The fundamental principle behind $\mathrm{CT}$, or image reconstruction from projections, has been known since the studies made by Radon [1] in 1917, in which the Radon inversion formula was derived and proved. CT methods have been used in many areas in recent years such as: application in soil science [2], study of porous structure of amorphous materials [3], etc. Since the presentation of the medical tomography, a great number of scanners have been projected and constructed $[4,5]$. Our project has some similarity from that proposed by Naime [6], since we use the same kind of radiation detection. In this work, we describe the project, construction and tests of a new low cost X-ray computerized tomography scanner for physicalchemical analysis and nondestructive general purpose inspections. Some images of different kinds of objects acquired with this scanner are showed and discussed. This facility is located at the Applied Nuclear Physics Laboratory of University of Sorocaba (LAFINAU) in Sorocaba, SP, Brazil. 


\section{MATERIALS AND METHODS}

In this section we present a complete description of the essentials parts of this X-ray CT facility. A microfocus X-ray source from Hamamatsu Corp. model L-673101 was the choice for our application, taking in to account technical specification and costs. L-6731-01 has a focal spot size of $8 \mu \mathrm{m}$ and produces a fan beam with an aperture angle of 39 degrees. This configuration is very appropriate to obtain image with high magnification and resolution. The X-ray source operates in a continuous mode and generates $\mathrm{X}$-rays with high stability. The target source is made of tungsten and operates with voltage between $20 \mathrm{kV}$ to $80 \mathrm{kV}$ and can supply X-rays with variable intensity by modifying the target current from $0 \mu \mathrm{A}$ to $100 \mu \mathrm{A}$. Several kinds of detectors can be employed for tomographic purpose, according to the various requirements, such as spatial and energy resolution, efficiency, cost, and so on. In the majority of tomographic projects, the radiation detectors become the principal cost. The aim was to construct a low cost and medium spatial resolution system to be used with objects from few millimeters to the size of around ten centimeters. Considering these facts, the S8865-128G commercial photodiode array (APD) from Hamamatsu Corp. was selected. With $300 \mu \mathrm{m} \times 600 \mu \mathrm{m}$ of dimensions and a tin layer of phosphor deposited at the entrance, the array is sensitive to X-rays in the energy range from 30 $\mathrm{keV}$ to $100 \mathrm{keV}$. This APD has an appropriate spatial resolution for the proposed applications, but it does not have any energy discrimination. To study large objects, a long line was constructed by connecting four APDs in a row one after the other. Each APD has 128 photodiodes arranged in $51.2 \mathrm{~mm}$ of length by $0.6 \mathrm{~mm}$ of height with each element diffusion presenting a width equals to $0.3 \mathrm{~mm}$. Thus, the final configuration of the radiation detector contains of 512 photodiodes, totalizing 204.8 $\mathrm{mm}$ of length. The signal generated by the PDAs was extracted using a driver circuit, also commercialized by Hamamatsu Corp. as model C9118-01. Figure 1a shows the experimental setup of our third-generation CT scanner.
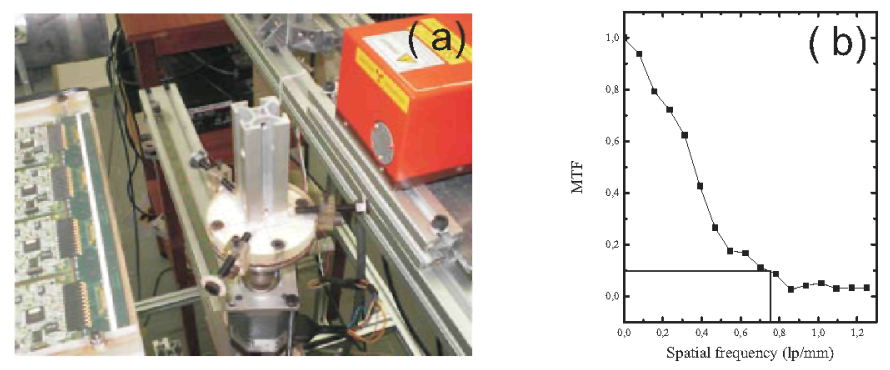

FIGURE 1. (a) Photograph of experimental LAFINAU X-ray CT Facility, where can be identified the following components: in the left side we see the PDAs, in centre of photograph is the sample and in the right side appear the X-ray source. Part (b) shows the MTF of this system

To control all equipments and data acquisition, a high-speed multifunction Mseries data acquisition (DAQ) PCI-6251 from National Instruments Corp. is used. A man-machine interface based on NI LabVIEW software was developed and used to control the data acquisition and other process. 


\section{IMAGE RECONSTRUCTION}

The mathematical algorithms for tomographic reconstruction are based on projection data. These projections can represent, for example, the linear attenuation coefficients of X-rays, along the path of the ray. The attenuation coefficients are dependent on the photon energy as well as on the density of objects or their constituents along the ray path. For the implemented solution, X-ray is considered an energy independent function. The most widely used reconstruction technique is called of Filtered Back Projection (FBP) method. Details on the theory of image reconstruction and practical algorithms may be found, for instance, in Ref. [7].

\section{RESULTS AND DISCUSSION}

The Modulation Transfer Function (MTF) is a main tool to evaluate the spatial resolution of an imaging system. In this work we use the method where a profile of the image of a cube across de ideal edge, provides de Edge Spread Function (ESF) of the system. The derivative of the ESF gives the Line Spread Function (LSF) of the system. The Fast Fourier Transform of a LSF furnishes the MTF. The MTF for the developed system presented in Figure $1 \mathrm{~b}$ exhibits, at $10 \%$ of MTF, a spatial resolution of 0.756 line pairs $/ \mathrm{mm}$ or $661 \mu \mathrm{m}$. The density resolution is used to evaluate the capability of $\mathrm{CT}$ to resolve small density differences. To analyze it, an image of a phantom showed in Figure 2a, composed by an aluminium cylinder, fulfilled with water and other objects inside then was used. Analyzing the image of this phantom, many color tonality can be discriminated, indicating the capability of the system in resolving different materials densities. The found values to the medium tomographic units measured in the points indicated in the Figure 2a are: Air (33 \pm 7 ), Water (54 \pm 13 ), Sand (105 \pm 12$)$, Cement (152 \pm 16$)$ and Aluminium (154 \pm 27$)$.

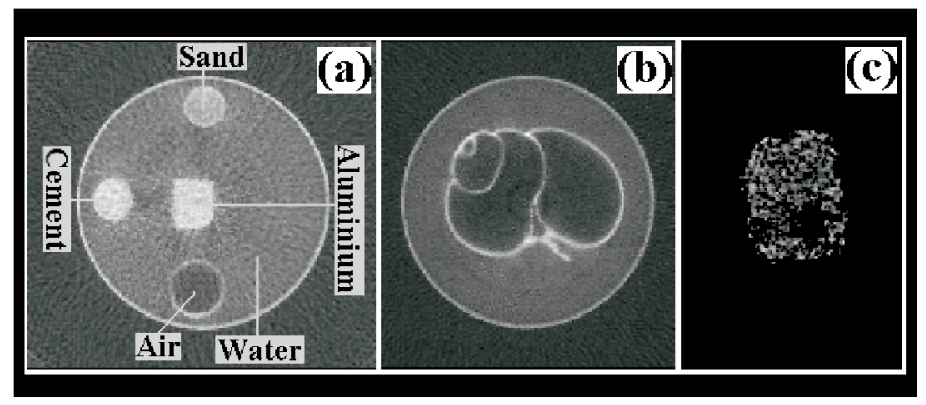

FIGURE 2. Part (a) shows an image of a phantom formed by an aluminium cylinder of $50 \mathrm{~mm}$ of diameter, fulfilled with water and other objects inside then. These objects are: three cylinder of aluminium fulfilled with cement; sand and air and one solid aluminium block, part (b) shows an image of a sea mollusk shell. The external circle is an aluminium recipient fulfilled with water and part (c) shows an image of a ceramic sample $(20 \mathrm{mmx} 15 \mathrm{~mm})$. The black areas in (c) are pores and the structures are shown by white areas. These images were reconstructed using FBP method. 
Figures $2 \mathrm{~b}$ and $2 \mathrm{c}$ show images of general applications. Figure $2 \mathrm{~b}$ shows $\mathrm{a}$ shell from a sea mollusk and the Figure 2c shows a piece $\mathrm{SiC}-\mathrm{Al}_{2} \mathrm{O}_{3}$ ceramic foam used for solid-fluid separation in metallurgical, automotive and petrochemical industries with porosity equals to $60 \mathrm{ppi}$ (pores per linear inch). This tomographic image can be compared with others, made using the same piece, but obtained by other group using a different tomographic system [3]. The resulted images are very similar indicating a good quality of the presented project.

\section{FINAL CONSIDERATIONS}

A low cost and medium resolution X-ray scanner for non-destructive testing application was projected, built and tested. The total cost of scanner was about of US\$ $35.000,00$ ( $12 \%$ of price of a similar equipment), what is very cheap if compared with the price of a similar commercial equipment. The system showed a spatial resolution of $661 \mu \mathrm{m}$ using a magnification factor equals to 2 . This resolution can be improved, if a larger magnification factor is used. The density resolution is good for an equipment that uses a system of detectors, with no energy discrimination. New tests are being made in order to find the best parameters to operate this tomographic system.

\section{ACKNOWLEDGMENTS}

The authors would like to thank the Fundação de Amparo à Pesquisa do Estado de São Paulo (FAPESP), Brazil, Grants 05/04727-6.

\section{REFERENCES}

1. J. Radon, "In the determination of functions from their integrals along certain manifolds", Berchte über die Verhandlugen, 69, 262-277 (1917).

2. P. E. Cruvinel, R. Cesareo, S. Crestana and S. Mascarenhas, "X-ray and gamma-ray computerized minitomograph scanner for soil science", IEEE Trans. Instrum. Meas., 39, (5), 745-750 (1990).

3. C. R. Appoloni, C. P. Fernandes, M. D. M. Innocentini, A. Macedo, "Ceramic Foams Porous Microstructure Characterization By X-ray Microtomography", Mat. Res., 7, (4), 557-564 (2004).

4. J. M. Oliveira Jr., "Project, Construction and Test of a Mini Computerized Tomograph", Braz. J. of Phys., 33, (2), 273-275 (2003).

5. U. Hampel, A. Bieberle, D. Hoppe, J. Kronenberg, E. Schleicher, T. Sühnel, F. Zimmermann and C. Zippe, "High resolution gamma ray tomography scanner for flow measurement and non-destructive testing applications", Rev. Scient. Inst. 78, (1) 103704-103704-9 (2007).

6. J. M. Naime, "Um Novo Método para Estudos Dinâmicos, In Situ, Da Infíltração Da Água Na Região Não-Saturada do Solo ", PhD. Thesis, Escola de Engenharia de São Carlos, da Universidade de São Paulo, 2001.

7. A. Kak and M. Slaney, "Principles of Computerized Tomographic Imaging", IEEE Press, New York, (1988). 
Copyright of AIP Conference Proceedings is the property of American Institute of Physics and its content may not be copied or emailed to multiple sites or posted to a listserv without the copyright holder's express written permission. However, users may print, download, or email articles for individual use. 